\title{
Gadolinium Deposition and the Patient's View on the Problem
}

$\mathbf{R}$ arely, radiology journals publish patients' perspectives concerning the gadolinium deposition. Therefore, the editorial by Mallio et $\mathrm{al}^{1}$ is unique, and I read it with great interest. In addition to mentioned facts, the following points are of great impact, too.

- The decision to inject gadolinium-based contrast agents (GBCAs) or not should be made, if possible, together with the patient. Wellinformed patients are able to choose the most suitable imaging procedure. Moreover, patient-centered care leads to satisfaction. ${ }^{2}$

- To realize the first point, the patient should be well informed. This means that the patient should be informed about the potential risk of gadolinium deposition in the brain and within several other organs and, as mentioned by Mallio et al, "patients should be informed that there is no documented clinical risk related to gadolinium deposition. ..." Moreover, patients should be also informed that delayed adverse events cannot be excluded.

- Symptoms self-reported by patients after exposure to GBCAs should be documented exactly. Currently, documentation of different adverse symptoms related to the application of contrast media is sparse or is missing. ${ }^{3}$ This is surprising because an exact documentation facilitated the everyday routine, as well as retrospective analyses.
- Patients with subjective symptoms should undergo a diagnostic procedure and should be closely monitored. To avoid overlooking harmful conditions, it is recommended to check the patient's symptoms.

Disclosures: Ingrid B. Böhm-UNRELATED: Travel/Accommodations/Meeting Expenses Unrelated to Activities Listed: Bayer AG Switzerland, Comments: German Radiology Congress (Leipzig) 2019.* *Money paid to the institution.

\section{REFERENCES}

1. Mallio CA, Quattrocchi CC, Rovira À, et al. Gadolinium deposition safety: seeking the patient's perspective. AJNR Am J Neuroradiol 2020;41:944-46 CrossRef Medline

2. Nairz K, Böhm I, Barbieri S, et al. Enhancing patient value efficiently: medical history interviews create patient satisfaction and contribute to an improved quality of radiologic examinations. PLoS One 2018;13: e0203807 CrossRef Medline

3. Böhm IB, van der Molen AJ. Recommendations for standardized documentation of contrast medium-induced hypersensitivity. J Am Coll Radiol 2020;17:1027-28 CrossRef Medline

(1) I.B. Böhm Department of Diagnostic, Interventional, and Pediatric Radiology, Inselspital University of Bern Bern, Switzerland 